OPEN ACCESS

Edited by:

Yilong Cheng,

Xi'an Jiaotong University, China

Reviewed by:

Ernesto Reverchon,

University of Salerno, Italy

He Liu,

Jilin University, China

*Correspondence:

Liqun Yang

yanglq@/nszjk.com.cn

Xinan Zhang

zhangxa2725@163.com

${ }^{\dagger}$ These authors have contributed equally to this work

Specialty section:

This article was submitted to Biomaterials,

a section of the journal

Frontiers in Materials

Received: 16 November 2021 Accepted: 04 January 2022

Published: 10 February 2022

Citation:

Zhang J, Wang W, Zhang X, Yang L and Zhang J (2022) Research Progress of Biodegradable Polymers in Repairing Achilles Tendon Injury.

Front. Mater. 9:815930 doi: $10.3389 /$ fmats.2022.815930

\section{Research Progress of Biodegradable Polymers in Repairing Achilles Tendon Injury}

\author{
Jinchi Zhang ${ }^{1,2+}$, Wange Wang $^{1 \dagger}$, Xinan Zhang ${ }^{1 *}$, Liqun Yang $^{2 *}$ and JinChao Zhang ${ }^{3}$ \\ ${ }^{1}$ College of Kinesiology, Shenyang Sport University, Shenyang, China, ${ }^{2}$ NHC Key Laboratory of Reproductive Health and Medical \\ Genetics (China Medical University) and Liaoning Key Laboratory of Reproductive Health, Liaoning Research Institute of Family \\ Planning (The Affiliated Reproductive Hospital of China Medical University), Shenyang, China, ${ }^{3}$ School of Physical Education, \\ Harbin Normal University, Harbin, China
}

Achilles tendon injury has become a common sports injury clinically, and its treatment and rehabilitation are essential, while the regenerative capacity of the Achilles tendon in adult mammals is limited. Therefore, it is necessary to promote the repair and remodelling of the Achilles tendon through efficient interventions. Biodegradable polymer materials are one of the most popular in the treatment and repair of soft tissues, ligaments, muscles, and organs injured by organisms to enhance the function of their wounded sites. Thus, it plays a specific role in "compensation" and is widely used in clinical medicine and rehabilitation. This review summarized the progress of poly ( $\varepsilon$-caprolactone), polylactic acid, poly (lacticco-glycolic acid), poly (trimethylene carbonate) (PTMC), and polydioxanone (PDS) in repairing Achilles tendon injury, indicating that the biodegradable polymers have succeeded in improving and treating Achilles tendon injuries. However, some problems such as lack of good affinity with cells and uncontrollable degradation of the biodegradable polymers should be overcome in repairing Achilles tendon injury. Therefore, the development of modified biodegradable polymers to make them an ideal repair material that meets the requirements is vital in improving Achilles tendon injuries. With the continuous development and close cooperation of life sciences and material sciences, excellent materials for repairing Achilles tendon injuries will undoubtedly be produced. The treatment of Achilles tendon injuries will be more straightforward, which will be a boon for many athletes.

Keywords: biodegradable polymers, Achilles tendon injury, poly ( $\varepsilon$-caprolactone), poly (lactic acid), poly (lactide-coglycolide), poly (trimethylene carbonate), polydioxanone

\section{INTRODUCTION}

The Achilles tendon is the largest and most powerful tendon in the human body, laying the foundation for our lower limb activities. Achilles tendon injury often occurs during the process of physical training. The damage of soft tissue and muscle ligaments around the Achilles tendon, causing the loss of lower limb muscle strength, flexibility, and other functions. If the Achilles tendon fracture is severe and surgical treatment is needed. Tendon injury is common in sports and other rigorous sports, leading to dysfunction, and disability for months or years (Dams et al., 2019). In addition to direct damage, more than $70 \%$ of the Achilles tendon breaks are more involved in sports, especially the ball and track and field (Ganestam et al., 2016). Complications after an Achilles tendon 
rupture are not uncommon. The tendon rupture was the most common. The postoperative Achilles tendon re-rupture rate was approximately 1.7-5.6\% (Cerrato and Switaj, 2017). The most common method of tendon injury is autologous transplantation. However, these lead to morbidity and further weakness (Jayasree et al., 2019).

In recent years, tissue engineering based on biomedical materials has given hope to repairing Achilles tendon injuries. Previous work showed that collagen is a popular scaffold material for tendon tissue engineering because tendons are principally composed of collagen (Garvin et al., 2003; Juncosa-Melvin et al., 2006). Although other naturally derived scaffolds researched for tendon applications have included chitosan-based hyaluronan hybrid polymer fibres (Funakoshi et al., 2005) and alginate-chitosan polyion hybrid fibrous complex (Majima et al., 2005), a significant focus has been targeted on synthetic degradable materials, such as PGA (Cao et al., 2006) and PLGA (Moffat et al., 2009). Revercho et al. had critically analyzed the biodegradable polyester like polyglycolic acid (PGA), polylactic acid (PLA), and their copolymer for tendon tissue engineering (Reverchon et al., 2012). They demonstrated the degradation products of polyesters, such as glycolic acid and lactic acid, are metabolites present in the human body, while the main disadvantage is that the degradation products are acidic and will cause inflammation. In addition, the hydrophobic structure of biodegradable polyesters may cause problems for cell adhesion. In contrast, poly ( $\varepsilon$-caprolactone) (PCL), polydioxanone (PDS), or poly (trimethylene carbonate) (PTMC) degrades with nonacidic products, avoiding the problem of $\mathrm{pH}$ drop. Hence, biodegradable polymers' selection and performance regulation will significantly impact the repair effect of Achilles tendon injury, which also attracts extensive interest.

In this review, the most widely studied biodegradable polymers, including polyesters, such as PLA and PLGA, and PTMC, PDS, are listed as representatives to summarize the progress in the application of biodegradable polymers in the repair of Achilles tendon injuries. It will provide detailed theoretical guidance for the selection and development of scaffold materials with good performance, and promote tissue recovery and regeneration of the Achilles tendon injury, making it a reality for patients to return to families and society healthily.

\section{EFFECT OF PCL ON THE REPAIR OF THE ACHILLES TENDON INJURY}

Poly ( $\varepsilon$-caprolactone) (PCL), has a highly effective therapeutic effect as a biomedical polymeric material combined with cells to treat Achilles tendon injuries. Cai et al. (2020) combined PCL-SF/ PLCL fabric scaffolds with marrow stem cells from rabbit bone to promote healing of injured tendons. It has been found that PCLSF/PLCL copolymer has good tissue regeneration ability. It will be a potentially valuable biomedical polymer material widely used in tendon tissue engineering.

It is worth noting that using a variety of biomedical polymers as composite scaffold materials can play a double role in repairing Achilles tendon injuries with half the effort. It was concluded that the Band-Aid with 50\% Wharton's jelly (WJ)-derived extracellular matrix (ECM) combined with PCL electrospinning had the fastest therapeutic effect on cell proliferation and differentiation, indicating that it was beneficial to nerve regeneration after Achilles tendon rupture and improved the activity of muscle tissue ( $\mathrm{Li}$ et al., 2017). Schoenenberger et al. (2018) prepared PCL fibre scaffolds with highly aligned or randomly oriented by electrospinning and cultured human tendon fibroblasts (TFs) on them. The results showed that highly aligned PCL scaffolds tended to downregulate the expression of matrix metalloproteinases (MMPs) because of their high catabolic activity; Czarnecki et al. (2012) similarly investigated the feasibility of PCL-modified composite fibre-carbon scaffolds and analyzed their mechanical properties and ability to support the growth and proliferation of human dermal fibroblasts. The results showed that PCL-modified composite fibrous scaffold had similar mechanical properties to the acellular dermal matrix to support fibroblast adhesion and proliferation. The results demonstrated that PCL, as the main component of the fibrous scaffold, can play a role in inhibiting cell adhesion and rapid proliferation physiologically and can be used as a biomedical polymer material in clinical studies.

In addition to the above composite fibre scaffold, the Achilles tendon can also be repaired directly using the interaction between biomaterials and cells. Wang has developed a bioactive polymer membrane for guided bone regeneration (GBR) by interacting bone marrow stromal cells (BMSCs) with PCL and functionalizing stromal cell-derived factor-1a (SDF-1a). The cultured biofilm can be applied to the injured bone and joint. The results suggest that SDF-1a-loaded PCL electrospun membranes are bioactive and valuable for optimizing the clinical application of GBR strategies (Ji et al., 2013).

To simulate the natural tendon tissue structure, Jayasree et al. (2019) prepared a woven multiscale fibrous scaffold composed of PCL micro/collagen bFGF nanofibers and coated with sodium alginate to prevent peritendinous adhesions. The release kinetics of bFGF showed sustained release of growth factors for 20 days. The results showed that mPCL-nCol-bFGF had higher cell proliferation and expression of tenogenic markers than mPCLnCol. The results also showed that PCL nanofibers scaffold loaded with essential fibroblast growth factor (bFGF) could recover some physiological indexes around the Achilles tendon of rabbit tendon. It could be used as a biomedical polymer material to repair Achilles tendon injury.

Chen et al. (2017) studied the physicochemical properties of random PCL (RP) nanofibers, random PCL/SF (RPSF) nanofibers, and aligned PCL/SF (APSF) nanofibers, and used RPSF and APSF scaffolds to repair rabbit Achilles tendon defects. Histological sections were stained with Hematoxylin-eosin (H\&E) and Masson's trichrome at 6 and 12 weeks. The results showed that the migration and proliferation of rabbit dermal fibroblasts (RDFBs) on the arranged nanofiber scaffolds tended to stretch in a parallel direction along with the arrangement of nanofibers, which was conducive to promoting soft tissue healing around Achilles tendon defects and improving the effectiveness and reliability of the scaffolds in vitro. In addition, scaffolds made of SF can also be applied to the regenerative repair of other tissues, 
for example, renewable bone, eye, nerve, skin, tendon, ligament, and cartilage (Jao et al., 2016).

As a biodegradable fibrous membrane, the biomedical polymer can make full use of its biological characteristics such as repair of Achilles tendon and rapid degradation rate, which is conducive to reducing the number of inflammatory factors around Achilles tendon injury can play a role in preventing soft tissue adhesion. Some experts have studied the applicability of PCL film for repairing the Achilles tendon gap in the rat model. The results showed that the biomechanical and morphological changes were similar in the PCL membrane repair Achilles tendinopathy group after 8 weeks of surgery (Kazimoğlu et al., 2003). It indicated that the biodegradable PCL membrane group had the best therapeutic effect on the Achilles tendon and demonstrated the practical therapeutic effect of PCL in repairing Achilles tendon injury.

It has been reported that Lee et al. (2019) also improved the healing of collagenase-induced Achilles tendinitis in rabbits by preparing diclofenac immobilized polycaprolactone (DFN/PCL) fibre sheets. The study results demonstrated that the long-term diclofenac delivery system using PCL fibre sheets strongly affects collagenase-induced Achilles tendinitis rabbit model and tendon recovery. By investigating the effect of PCL ultrafine fibres arranged in three dimensions on the growth behaviour of fibroblasts, it was found that microfibers were able to support the proliferation of human dermal fibroblasts for more than 7 days (An et al., 2012). This super fibre can be considered a scaffold study for applying biomedical polymer materials in tissue engineering and will be an effective clinical treatment option for athletes with Achilles tendon injuries.

Others have combined composite fibrous materials with stem cells to prepare new biomedical polymer materials to promote soft tissue regeneration around the Achilles tendon, enhance cell activity, and avoid pathological changes in the injured tendon tissue. Dong et al. (2021) found that a biopolymer scaffold composed of polycaprolactone/silk fibroin (PCL/SF) copolymers could reduce the adhesion of tissues around the Achilles tendon, thereby promoting the healing of Achilles tendon injuries. Bosworth et al. (2013) used three different polycaprolactone fibre-based electrospinning scaffolds (twodimensional random sheets, two-dimensional alignment sheets, and three-dimensional bundles.) to repair injured tendons. The results showed that the three-dimensional bundle showed the most excellent tensile performance, significantly stiffer than the two-dimensional arrangement and two-dimensional random fibres. It can be used as a biomaterial for artificial grafts, and considered the polymer material of choice to treat Achilles tendon ruptures.

The study of Achilles tendon reconstruction is a hot topic. Immunohistochemical and biomechanical analysis of the tendon regeneration process using braided tendon implants for Achilles tendon reconstruction in rabbits showed that both polylactic acid and chitin composite tendons had good initial strength and increased fibrous tissue length. It also plays a compliant role in reconstructing the Achilles tendon, indicating that these polymer materials have great potential in artificial tendons (Sato et al., 2000). Hu et al. (2013) prepared PCL biofilms using an improved melt moulding/leaching technique and evaluated their physical and mechanical properties and in vitro degradation rates, while biomechanical analysis was carried out after repairing the Achilles tendon of rabbits after repair rupture. The results showed that the internal fixation technique of strengthening tendon repair with PCL biofilm could significantly improve the tensile strength of the Achilles tendon repair site, which laid a foundation for early postoperative rehabilitation.

As mentioned above, PCL has good biocompatibility and has great application potential in repairing Achilles tendon injuries as one of the ideal repair materials. However, PCL is a semicrystalline polymer; its degradation rate in vivo is prolonged and affected by crystallinity. The higher the crystallinity, the slower the degradation. How to adjust the all-around performance of PCL, make it have a controllable degradation rate to match the degradation rate with the speed of tissue regeneration is a problem that needs to be addressed in the application of PCL to repair Achilles tendon injuries. In addition, whether the crystalline degradation fragments of PCL can induce inflammation is also a question worthy of attention.

\section{EFFECT OF PLA ON ACHILLES TENDON REPAIR}

Polylactic acid (PLA) has been widely used in regenerative medicine as an absorbable, biodegradable polymer. Due to its excellent biological characteristics, PLA has also attracted more and more attention in repairing Achilles tendon injuries. Liu et al. (2015) produced the electrospun PLA nanofibers with controllable coiling. Studies have shown that electrospinning PLA has controllability and can play a therapeutic effect in restoring tendon injury. It is common to prepare PLA composite scaffold materials to repair Achilles tendon injury, as coupled with the intervention of various cytokines, the regeneration of the Achilles tendon and the proliferation and differentiation function cells will be promoted. Vuornos et al. (2016) reported that human adipose-derived stem cells (hASCs) could form a uniform cell layer on woven PLA scaffolds under the culture of a tenogenic medium, and effectively differentiate into tendon tissue.

Unlike the fibrous scaffolds loaded with hASCs described above, Wang et al. (2017) compared the effect of cell-free scaffolds, allogeneic scaffolds and autologous cell implantation scaffolds on the tissue recovery. The allogeneic scaffold material was PGA with PLA fibres woven in a ratio of 4:2. The study results found that the degree of tissue recovery in the allogeneic group was better than the other two groups, and the degradation rate of the scaffold in vivo was much faster; Deng et al. (2014) also used a composite tendon scaffold consisting of a PGA unwoven fibre medial and a PGA/PLA wool woven mesh lateral to provide mechanical strength. The results showed that adipose-derived stem cells (ASCs) combined with biomaterials could rapidly heal the ruptured tendon, produce new granulation tissue, and gradually form mature new tendons. The results indicate that 
ASCs have a huge promoting effect in tissue remodelling and tendon regeneration.

In addition to the above composite scaffold materials, PLA also can be used to prepare new hollow braided prosthetic material that can be degraded in vivo to repair Achilles tendon injury. Araque-Monrós et al. (2013) designed a new renewable absorbable tendon and ligament prosthesis through PLA hollow braided bands, finfding that L929 lineage fibroblasts combined with PLA braid could prevent tissue adhesion and promote the continuous proliferation and renewal of cells. Chen et al. (2009) reported that the regeneration limitations of injured biological tissues have been solved by using the characteristics of cells in combination with material scaffolds for tendon and ligament injuries.

PLA has good biological characteristics, will not have adverse effects on the body, and plays an essential role in repairing Achilles tendon injury. Song et al. (2015a) used PLA and PCL electrospinning membranes with different degradation kinetics to study the anti-adhesion effect on Achilles tendon repair. The results showed that the electrospun PLA membrane group's antiadhesion ability and tendon repairability were significantly better than those of the PCL membrane group.

At present, Achilles tendon rupture requires the fixation of an external splint, and the traditional sling will cause muscle spasms. Blaya F et al. prepared splints made of made of PLA and FilaFlex to ensure comfort and corrosion resistance (Blaya et al., 2019). Some researchers have prepared anti-adhesion films by co-spinning beeswax (Wax) with PLA. In the rat Achilles tendon adhesion model, the beeswax/PLA film group had the least histologically surrounding adhesions at the repair site, which indicated that the anti-adhesive effect of beeswax/ PLA copolymer on the Achilles tendon was significant and could be considered to prevent the adhesion of Achilles tendon soft tissue (Zou et al., 2020).

From autologous transplantation to "compensatory" replacement of grafts with medical polymer materials, this will be a significant advance in applying medical polymer materials, but the histocompatibility of this biomedical polymer material to the human body, and whether there will be eosinophilic substances need to be further studied. To promote the broad application of PLA in the repair of Achilles tendon injuries, eliminating the inflammation caused by acid degradation products is a critical issue that needs attention. Another issue that needs to be considered is how to increase the affinity of PLA and cells to improve cell adhesion and proliferation viability.

\section{EFFECT OF PLGA ON ACHILLES TENDON REPAIR}

The scaffold materials composed of PLGA combined with cells are also reoprted in Achilles tendon injury. For example, the complex of stem cells (MSCs), fibrin glue, and woven PLGA to repair the tendon defects of gastrocnemius tendon and patella have shown that the tensile modulus of the gastrocnemius tendon reaches $62 \%$ of normal tissue 12 weeks after implantation (Awad et al., 2003; Ouyang et al., 2003).
It is a relatively novel tissue engineering method to encapsulate the bioactive substance on reinforced degradable knitted fibres, combined with cellular components for repairing Achilles tendon injuries. Sahoo et al. (2010) encapsulated bFGF on a slowly degrading knitted PLGA microfiber filament scaffold to fabricate a new biohybrid fibre scaffold system. It has been found that this hybrid polymer scaffold can promote the attachment and proliferation of intercellular progenitor cells (MPC) and allow the cells to grow on PLGA fibre scaffolds, fully indicating that PLGA is very potential for repairing and regenerating tendons; Ouyang et al. (2002) reported the application of woven PLGA scaffold seeded with bone marrow stromal cells (bMSC) in improving Achilles tendon injury in rabbits. bMSC/PLGA-treated tendon repair had eosinophilic tissue formation after stent implantation, which indicated that this biological scaffold produced cell proliferation and regeneration in the soft tissue around the Achilles tendon, resulting in treatment around the Achilles tendon injury. However, acidic substances will produce an acidic environment around the Achilles tendon, which is not conducive to the healing of the Achilles tendon.

Some experts prepared silk fibroin-PLGA mesh scaffolds, filling with type I collagen and rabbit autologous bone marrow stem cells. Implantation of these scaffolds into rabbit Achilles tendon defect sites resulted in the formation of granulation tissue exhibited by the regenerated Achilles tendon, indicating that necrotic tissue was being repaired and inflammatory factors were reduced (Zhang et al., 2015). Choi et al. (2020) prepared lactoferrin-immobilized, heparinanchored PLGA nanoparticles and delivered them to rat Achilles tendon sites with Achilles tendinitis. The collagen and factor around the tendon increased, showing that PLGA nanoparticles have an excellent therapeutic effect on Achilles tendinitis.

Yan et al. (2021) concluded that fibroblast growth factor (bFGF) can promote tendon healing, and they prepared PLGA electrospun membrane loaded with ibuprofen (IBU) and bFGF to treat Achilles tendon injuries in animals. The study showed that the PLGA electrospun film loaded with bFGF and IBU significantly reduced inflammatory factors and increased collagen fibres at the Achilles tendon healing area. Weng et al. (2020) studied the use of electrospinning to develop antibiotics loaded ultrafine materials. It was found that PLGA nanofibers released effective concentrations of epinomycin more than 40 days after surgery. At 1.5 weeks, the maximum intensity levels of control tendons were lower than that of healthy tendons. At 3 weeks, the doxycycline group showed a top tendon strength level comparable to the healthy tendon group. The control group did not receive a doxycycline nanofiber membrane, and the maximum intensity was poor compared to the other two groups. Six weeks after surgery, the tendon strength was comparable in all three groups, while the values in the doxycycline group were slightly more significant than the other groups, and the rats also showed better mobility and stronger tendons after surgery.

In addition to using sustained-release systems, some scholars have used new bioactive scaffolds to transplant multifunctional stem cells to repair Achilles tendon injury. Zhao et al. (2019) integrated MSC sheets and bFGF into PLGA/bFGF-fibrin gel 
scaffolds, promoting the proliferation, and differentiation of MSCs into tendons and synergistically promoting the reconstruction of injured tendons. Histological observation of regenerated tendons at 8 weeks after transplantation showed that the PLGA/bFGF-fibrin gel scaffold integrated with MSC sheets and bFGF had more interwoven collagen fibres compared with the PLGA/MSCs and PLGA/bFGF groups, while the control group had a lower content of fibrocytes and myofibers. Therefore, the transplantation of PLGA/MSCs/bFGF into injured tendon sites in humans can promote the remodelling of necrotic cells around tendons, improve the degree of tendon injury, and promote the repair and regeneration of tendons.

It is a novel idea to use PLGA hydrogel as a carrier in tissue engineering to repair Achilles tendon injury. The use of hydrogel can improve the body's compatibility and promote the removal of necrotic tissue. Yuan et al. (2015) injected 5-Fuloaded PLGA-PEG-PLGA hydrogel into the severed Achilles tendon on the right side of the rat. Studies have shown that 5-Fu-loaded PLGA hydrogel can effectively inhibit the adhesion and contracture of Achilles tendon soft tissue, providing a solid guarantee for clinical research of Achilles tendon injury. Similarly, the unit model treated with PLGA film prepared by electrospinning also showed better inhibition of tissue adhesion and tissue repair ability (Song et al., 2015b).

Making appropriate improvements in the performance of biomedical polymer materials and combining various cells to repair Achilles tendon injuries will improve the repair efficiency of Achilles tendon injuries. El Khatib et al. (2020) determined the biological characteristics of sheep amniotic epithelial cells by changing time and distance by treating PLGA with highly aligned microfibers using cold atmospheric plasma (CAP). The results showed that the hydrophilicity and cellular characteristics of PLGA microfibers were improved by treating PLGA microfibers with CAP in the range of $1.3 \mathrm{~m}$.

In addition to the use of biomedical polymer materials, the combined use of drugs for the intervention of Achilles tendon injury will maximize its repair function. Chen et al. (2020) prepared Collagen Hybridizing Peptide (CHP)-modified PLGA nanoparticles and delivered them to mouse Achilles tendons using rapamycin (RAPA) as a drug model. The results showed that CHP-PLGA-RAPA nanoparticles reflected histocompatibility and affinity when repairing Achilles tendon injuries, allowing rapid repair of injured Achilles tendon tissues.

Although numerous studies have shown that PLGA is a promising material for repairing Achilles tendon injuries, the production of acid degradation products and the lack of active groups that interact with cells are still the main factors limiting its wide application in the repair of Achilles tendon injury. Blending or graft modification with natural polymers may be an effective strategy to solve the above problems, which will inevitably become a research hotspot in this field.

\section{EFFECT OF PTMC ON ACHILLES TENDON REPAIR}

Poly (trimethylene carbonate) (PTMC) is formed by ringopening condensation of trimethylene carbonate monomer, which has non-toxicity, good cytocompatibility, and degradability.

Li et al. (2020) studied nanofibrous PCL/PTMC-MA biopolymer scaffolds. The results showed that the mechanical properties of polymer composite scaffolds composed of PTMC combined with PCL increased significantly, which prevented tissue adhesion and promoted cell proliferation and differentiation.

Shieh et al. (1990) prepared polymer material fibres from random copolymers of 90\% DMTMC and 10\% TMC. They retain excellent biomechanical properties and have appropriate biodegradability. It lays a foundation for the proliferation and migration of rabbit Achilles tendon cells and further improves the activity of stromal cells. Duek et al. (2014) used P (LDLA-TMC) membrane as a protective membrane and implanted it into adjacent tissues in New Zealand rabbits to prevent their adhesion. The results showed that the proliferation and differentiation of cells were accompanied by scar formation around the soft tissue, indicating that the $\mathrm{P}$ (LDLA-TMC) membrane has a specific anti-adhesion effect in repairing Achilles tendon injury.

PTMC does not produce acidic degradation products during in vivo degradation, allowing a reduction in the number of inflammatory factors around the Achilles tendon and removing necrotic scar tissue. Although PTMC overcomes the disadvantages of producing eosinophilic substances, its application in repairing Achilles tendon injuries is less studied, and there are still some problems that need to be solved urgently. For example, further study is required to ensure that stem cells can play a full role in PTMC scaffold materials and are evenly distributed on the material surface without shedding.

\section{EFFECT OF PDS ON ACHILLES TENDON REPAIR}

Polydioxanone (PDS) has non-toxicity, contamination, complete degradation, and good mechanical properties. Using PDS loaded fibroblasts to suture the Achilles tendon injury site can promote fibroblasts to exert their unique properties and heal Achilles tendon injury. He et al. (2002) seeded fetal skin fibroblasts into the human amniotic extracellular matrix (HA-ECM) and wrapped them around the Achilles tendon injury suture sutured by PDS. The immunohistochemical results showed that the labelled seed fibroblasts grew well. The rate and quality of Achilles tendon recombination in the experimental group were better than those in the control group, indicating the use of PDS is used helpful for the repair of Achilles tendon injury.

Unlike the above studies, Moshiri et al. (2015) fabricated a collagen implant $(\mathrm{CI})$ as a simulated tendon, simulated the accessory tendon with a PDS sheath, and then embedded bovine platelet gel (BPG) as an active source of growth factors in rabbits Achilles tendon defects. Studies have found that activated platelets in the scaffold release significantly higher growth factors than the control group, and the turnover of peritendinous fibrocytes and remodelling of the Achilles 
tendon have also been further improved. Oryan et al. (2014) also studied the effect of 3D collagen and collagen/PDS implantation on rabbit Achilles tendon defect model by observing the histopathology and ultrastructural structure of injured Achilles tendon and intact Achilles tendon. It has been shown that the implantation of 3D collagen and collagen/PDS accelerates the production of new tendons in the defect area and restores the average functional level of the Achilles tendon.

PDS has excellent mechanical and mechanical properties, playing a role in consolidating acute Achilles tendon rupture in humans. Gebauer et al. (2007) conducted a comparative experiment of the surgical suture in patients with acute Achilles tendon. The results showed that the Bunnell method had primary suture stability than the Kessler method, and PDS had higher suture strength. It indicates that PDS as a suture material can help connect the broken end of the human Achilles tendon and play a role in fixation and support. Ji et al. (2015) compared the effect of PDSII and Ethibond W4843 surgical sutures in treating acute Achilles tendon rupture. By postoperative observation, the two suture biomedical materials had an almost consistent therapeutic effect in incision infection and re-rupture rate, but in the follow-up score, the wound healing effect of PDSII was slightly inferior to Ethibond. Yildirim et al. (2006) found that the two PDS sutures had the highest tendon stress, indicating that PDS has the loading capacity and excellent elastic properties, plays a tight junction role in the healing of fresh Achilles tendon, and provides practical help for the tissue healing and cell regeneration of Achilles tendon rupture.

Some scholars have also reported that using PDS and nonuse of PDS as carrier substances for repairing the Achilles tendon will have different therapeutic effects. MeimandiParizi et al. (2013) used collagen and collagen-PDS implants to reconstruct Achilles tendon defects. Studies have shown that with the introduction of PDS materials, the biomechanical properties of the experimental group have been significantly improved, the necrotic tissue around the injured tendon has been reduced, and the soft tissue adhesion around the Achilles tendon has also been effectively inhibited.

In summary, PDS has the advantages of good degradability and biocompatibility and is now widely used in the field of clinical medicine, such as surgical sutures, which can provide vital help for the future of Achilles tendon rupture and promote the rapid recovery of the mechanical characteristics and functional level of the Achilles tendon.

\section{EFFECT OF OTHER MATERIALS ON ACHILLES TENDON REPAIR}

Pietschmann et al. (2013) prepared Polyglycolic acid (PGA) scaffolds for bridge Achilles tendon rupture in rats. The study results showed that the application of MSC significantly improved the healing rate of the Achilles tendon compared with the PGA scaffold alone group.
Nguyen et al. (2020) studied the effect of polyethene (PE) sutures in repairing human Achilles tendon rupture and evaluated the mechanical properties of the suture. The results showed that PE could be used as a medical polymer material to suture human Achilles tendon rupture, but the tensile resistance and degradability are general.

Cai et al. (2018) seeded allogeneic bone marrow stem cells (BMSCs) were on polyethylene terephthalate (PET) scaffolds and then implanted into rabbit unilateral Achilles tendon defects. The results showed that 12 weeks after surgery, the BMSCs-PET group was significantly more substantial than the control PET group in terms of type I and III collagen fibres and mechanical characteristics. In addition, researchers have simulated the fibrous structure of Achilles tendons with different structures of polypropylene (PP) and PET, and PET Achilles tendons showed some anti-fatigue and appropriate creep characteristics (Morais et al., 2020). Unlike the above studies, Gall et al. (2009) investigated the mechanical stability of polypropylene (PP) shapes in repairing distal Achilles tendon ruptures in dogs. The results showed that PP could be used as a medical polymer material to repair the Achilles tendon after surgery.

\section{CONLUSION}

The ideal scaffold material is still a critical issue for tendon tissue engineering. The research results of scaffold materials will directly promote the development of tendon tissue engineering and have broad clinical application prospects. In this review, we summarized the progress of biodegradable polymers, such as PCL, PLA, PLGA, PTMC, and PDS, in treating Achilles tendon injury. The biodegradable polymers loaded with stem cells or drugs can significantly improve the Achilles tendon injury repair effect. Although the research and application of scaffold materials derived from biodegradable polymers in tendon tissue engineering have succeeded, the currently used materials may have biocompatibility and degradability problems or have defects such as poor mechanical properties complex in processing and moulding. There are still some problems in applying biodegradable polymers to repair Achilles tendon injury that needs to be further explored; for example, are the adverse effects of biodegradable polymers on human function significant? Can the patient be helped to maximize functional recovery? Can tissue-engineered scaffold materials produce relatively ideal therapeutic results in humans and animal models? Are there differences in physiological and biomechanical properties between regenerated and native Achilles tendons? The above problems still need further study to be solved. Therefore, developing synthetic materials and modified natural materials to make them an ideal scaffold material that meets the requirements is an essential direction of repairing Achilles tendon injury. With the continuous development and close cooperation of life sciences and material sciences, ideal materials for repairing Achilles tendon injuries will undoubtedly be produced. The treatment of Achilles tendon 
injuries will be more straightforward, which will be a boon for many athletes.

\section{AUTHOR CONTRIBUTIONS}

$\mathrm{JZ}$ and WW wrote this article and equally contributed to this work; JCZ revised this article; XZ and LY checked and review this article.

\section{REFERENCES}

An, J., Chua, C. K., Leong, K. F., Chen, C.-H., and Chen, J.-P. (2012). Solventfree Fabrication of Three Dimensionally Aligned Polycaprolactone Microfibers for Engineering of Anisotropic Tissues. Biomed. Microdevices 14 (5), 863-872. doi:10.1007/s10544-012-9666-3

Araque-Monrós, M. C., Gamboa-Martínez, T. C., Santos, L. G., Bernabé, S. G., Pradas, M. M., and Estellés, J. M. (2013). New Concept for a Regenerative and Resorbable Prosthesis for Tendon and Ligament: Physicochemical and Biological Characterization of PLA-Braided Biomaterial. J. Biomed. Mater. Res. A. 101 (11), 3228-3237. doi:10.1002/jbm.a.34633

Awad, H. A., Boivin, G. P., Dressler, M. R., Smith, F. N. L., Young, R. G., and Butler, D. L. (2003). Repair of Patellar Tendon Injuries Using a CellCollagen Composite. J. Orthop. Res. 21 (3), 420-431. doi:10.1016/s07360266(02)00163-8

Blaya, F., Pedro, P. S., Pedro, A. B. S., Lopez-Silva, J., Juanes, J. A., and D'Amato, R. (2019). Design of a Functional Splint for Rehabilitation of Achilles Tendon Injury Using Advanced Manufacturing (AM) Techniques. Implementation Study. J. Med. Syst. 43 (5), 122-215. doi:10.1007/s10916019-1247-z

Bosworth, L. A., Alam, N., Wong, J. K., and Downes, S. (2013). Investigation of 2D and 3D Electrospun Scaffolds Intended for Tendon Repair. J. Mater. Sci. Mater. Med. 24 (6), 1605-1614. doi:10.1007/s10856-013-4911-8

Cai, J., Yang, Y., Ai, C., Jin, W., Sheng, D., Chen, J., et al. (2018). Bone Marrow Stem Cells-Seeded Polyethylene Terephthalate Scaffold in Repair and Regeneration of Rabbit Achilles Tendon. Artif. Organs 42 (11), 1086-1094. doi:10.1111/aor.13298

Cai, J., Xie, X., Li, D., Wang, L., Jiang, J., Mo, X., et al. (2020). A Novel Knitted Scaffold Made of Microfiber/nanofiber Core-Sheath Yarns for Tendon Tissue Engineering. Biomater. Sci. 8 (16), 4413-4425. doi:10.1039/ d0bm00816h

Cao, D., Liu, W., Wei, X., Xu, F., Cui, L., and Cao, Y. (2006). In Vitro tendon Engineering with Avian Tenocytes and Polyglycolic Acids: a Preliminary Report. Tissue Eng. 12 (5), 1369-1377. doi:10.1089/ten.2006.12.1369

Cerrato, R., and Switaj, P. (2017). Using Arthroscopic Techniques for Achilles Pathology. Foot Ankle Clin. 22 (4), 781-799. doi:10.1016/j.fcl.2017.07.007

Chen, J., Xu, J., Wang, A., and Zheng, M. (2009). Scaffolds for Tendon and Ligament Repair: Review of the Efficacy of Commercial Products. Expert Rev. Med. Devices 6 (1), 61-73. doi:10.1586/17434440.6.1.61

Chen, C.-H., Chen, S.-H., Kuo, C.-Y., Li, M.-L., and Chen, J.-P. (2017). Response of Dermal Fibroblasts to Biochemical and Physical Cues in Aligned Polycaprolactone/silk Fibroin Nanofiber Scaffolds for Application in Tendon Tissue Engineering. Nanomaterials 7 (8), 219. doi:10.3390/nano7080219

Chen, Y., Shen, W., Tang, C., Huang, J., Fan, C., Yin, Z., et al. (2020). Targeted Pathological Collagen Delivery of Sustained-Release Rapamycin to Prevent Heterotopic Ossification. Sci. Adv. 6 (18), eaay9526. doi:10.1126/ sciadv.aay 9526

Choi, H. J., Choi, S., Kim, J. G., Song, M. H., Shim, K.-S., Lim, Y.-M., et al. (2020). Enhanced Tendon Restoration Effects of Anti-inflammatory, Lactoferrin-Immobilized, Heparin-Polymeric Nanoparticles in an Achilles Tendinitis Rat Model. Carbohydr. Polym. 241, 116284. doi:10.1016/j.carbpol.2020.116284

Czarnecki, J. S., Lafdi, K., Joseph, R. M., and Tsonis, P. A. (2012). Hybrid Carbon-Based Scaffolds for Applications in Soft Tissue Reconstruction. Tissue Eng. Part. A. 18 (9-10), 946-956. doi:10.1089/ten.TEA.2011.0533

\section{FUNDING}

This work was supported by the Liaoning Revitalization Talents Program (XLYC1807142), the Department of Science and Technology of Liaoning Province (2018225079), the Educational Department of Liaoning Province (ZF2019040), and the Shenyang Science and Technology Bureau (RC190426).

Dams, O. C., van den Akker-Scheek, I., Diercks, R. L., Wendt, K. W., Zwerver, J., and Reininga, I. H. F. (2019). Surveying the Management of Achilles Tendon Ruptures in the Netherlands: Lack of Consensus and Need for Treatment Guidelines. Knee Surg. Sports Traumatol. Arthrosc. 27 (9), 2754-2764. doi:10.1007/s00167-018-5049-5

Deng, D., Wang, W., Wang, B., Zhang, P., Zhou, G., Zhang, W. J., et al. (2014). Repair of Achilles Tendon Defect with Autologous ASCs Engineered Tendon in a Rabbit Model. Biomaterials 35 (31), 8801-8809. doi:10.1016/j.biomaterials.2014.06.058

Dong, L., Li, L., Song, Y., Fang, Y., Liu, J., Chen, P., et al. (2021). MSC-derived Immunomodulatory Extracellular Matrix Functionalized Electrospun Fibers for Mitigating Foreign-Body Reaction and Tendon Adhesion. Acta Biomater. 133, 280-296. doi:10.1016/j.actbio.2021.04.035

Duek, J. R., Riquetto, M. L., Jesus, D. C., Sabongi, J. J., Barbo, M. d. L. P. B., Duek, E. A. d. R., et al. (2014). Membrana de PLDLA-TMC como Protetor na Regeneração Do Tendão Calcâneo. Estudo In Vivo em Coelhos. Polímeros 24 (3), 360-366. doi:10.4322/polimeros.2014.021

El Khatib, M., Mauro, A., Wyrwa, R., Di Mattia, M., Turriani, M., Di Giacinto, O., et al. (2020). Fabrication and Plasma Surface Activation of Aligned Electrospun PLGA Fiber Fleeces with Improved Adhesion and Infiltration of Amniotic Epithelial Stem Cells Maintaining Their Teno-Inductive Potential. Molecules 25 (14), 3176. doi:10.3390/molecules 25143176

Funakoshi, T., Majima, T., Iwasaki, N., Suenaga, N., Sawaguchi, N., Shimode, K., et al. (2005). Application of Tissue Engineering Techniques for Rotator Cuff Regeneration Using a Chitosan-Based Hyaluronan Hybrid Fiber Scaffold. Am. J. Sports Med. 33 (8), 1193-1201. doi:10.1177/ 0363546504272689

Gall, T. T., Santoni, B. G., Egger, E. L., Puttlitz, C. M., and Rooney, M. B. (2009). In Vitro biomechanical Comparison of Polypropylene Mesh, Modified Three-Loop Pulley Suture Pattern, and a Combination for Repair of Distal Canine Achilles' Tendon Injuries. Vet. Surg. 38 (7), 845-851. doi:10.1111/j.1532-950x.2009.00598.x

Ganestam, A., Kallemose, T., Troelsen, A., and Barfod, K. W. (2016). Increasing Incidence of Acute Achilles Tendon Rupture and a Noticeable Decline in Surgical Treatment from 1994 to 2013. A Nationwide Registry Study of 33,160 Patients. Knee Surg. Sports Traumatol. Arthrosc. 24 (12), 3730-3737. doi:10.1007/s00167-015-3544-5

Garvin, J., Qi, J., Maloney, M., and Banes, A. J. (2003). Novel System for Engineering Bioartificial Tendons and Application of Mechanical Load. Tissue Eng. 9 (5), 967-979. doi:10.1089/107632703322495619

Gebauer, M., Beil, F. T., Beckmann, J., Sárváry, A. M., Ueblacker, P., Ruecker, A. H., et al. (2007). Mechanical Evaluation of Different Techniques for Achilles Tendon Repair. Arch. Orthop. Trauma Surg. 127 (9), 795-799. doi:10.1007/s00402-007-0325-8

He, Q., Li, Q., Chen, B., and Wang, Z. (2002). Repair of Flexor Tendon Defects of Rabbit with Tissue Engineering Method. Chin. J. Traumatol. 5 (4), 200-208.

Hu, J. Z., Zhou, Y. C., Huang, L. H., and Lu, H. B. (2013). Development of Biodegradable Polycaprolactone Film as an Internal Fixation Material to Enhance Tendon Repair: an In Vitro Study. BMC Musculoskelet. Disord. 14 (1), 246-248. doi:10.1186/1471-2474-14-246

Jao, D., Mou, X., and Hu, X. (2016). Tissue Regeneration: a Silk Road. J. Funct. Biomater. 7 (3), 22. doi:10.3390/jfb7030022

Jayasree, A., Kottappally Thankappan, S., Ramachandran, R., Sundaram, M. N., Chen, C.-H., Mony, U., et al. (2019). Bioengineered Braided Micronano (Multiscale) Fibrous Scaffolds for Tendon Reconstruction. ACS Biomater. Sci. Eng. 5 (3), 1476-1486. doi:10.1021/acsbiomaterials.8b01328 
Ji, W., Yang, F., Ma, J., Bouma, M. J., Boerman, O. C., Chen, Z., et al. (2013). Incorporation of Stromal Cell-Derived Factor-1a in PCL/gelatin Electrospun Membranes for Guided Bone Regeneration. Biomaterials 34 (3), 735-745. doi:10.1016/j.biomaterials.2012.10.016

Ji, Y., Ma, X., Wang, X., Huang, J., Zhang, C., and Chen, L. (2015). Different Sutures in the Surgical Treatment of Acute Closed Achilles Tendon Rupture. Indian J. Surg. 77 (3), 936-940. doi:10.1007/s12262-014-1068-8

Juncosa-Melvin, N., Boivin, G. P., Galloway, M. T., Gooch, C., West, J. R., and Butler, D. L. (2006). Effects of Cell-To-Collagen Ratio in Stem Cell-Seeded Constructs for Achilles Tendon Repair. Tissue Eng. 12 (4), 681-689. doi:10.1089/ten.2006.12.681

Kazimoğlu, C., Bölükbaşi, S., Kanatli, U., Senköylü, A., Altun, N. S., Babaç, C., et al. (2003). A Novel Biodegradable PCL Film for Tendon Reconstruction: Achilles Tendon Defect Model in Rats. Int. J. Artif. Organs 26 (9), 804-812.

Lee, T. H., Kim, S. E., Lee, J. Y., Kim, J. G., Park, K., and Kim, H.-J. (2019). Wrapping of Tendon Tissues with Diclofenac-Immobilized Polycaprolactone Fibrous Sheet Improves Tendon Healing in a Rabbit Model of Collagenase-Induced Achilles Tendinitis. J. Ind. Eng. Chem. 73, 152-161. doi:10.1016/j.jiec.2019.01.018

Li, P. H., Liu, S. Y., Huang, J. X., Peng, J., Lu, S., and Guo, Q. (2017). In Vitro preparation and Characterization of Dual Biomimetic Electrospun Wharton's Jelly-Derived Extra Cellular Matrix/polycaprolactone Submicron Fibrous Band-Aid for superior Achilles Tendon Recovery. Int. J. Clin. Exp. Med. 10, 11563-11573.

Li, X., Chen, H., Xie, S., Wang, N., Wu, S., Duan, Y., et al. (2020). Fabrication of Photo-Crosslinkable Poly(Trimethylene Carbonate)/Polycaprolactone Nanofibrous Scaffolds for Tendon Regeneration. Int. J. Nanomedicine 15, 6373-6383. doi:10.2147/ijn.s246966

Liu, W., Lipner, J., Moran, C. H., Feng, L., Li, X., Thomopoulos, S., et al. (2015). Generation of Electrospun Nanofibers with Controllable Degrees of Crimping through a Simple, Plasticizer-Based Treatment. Adv. Mater. 27 (16), 2583-2588. doi:10.1002/adma.201500329

Majima, T., Funakosi, T., Iwasaki, N., Yamane, S.-T., Harada, K., Nonaka, S., et al. (2005). Alginate and Chitosan Polyion Complex Hybrid Fibers for Scaffolds in Ligament and Tendon Tissue Engineering. J. Orthopaedic Sci. 10 (3), 302-307. doi:10.1007/s00776-005-0891-y

Meimandi-Parizi, A., Oryan, A., and Moshiri, A. (2013). Tendon Tissue Engineering and its Role on Healing of the Experimentally Induced Large Tendon Defect Model in Rabbits: a Comprehensive In Vivo Study. PloS one 8 (9), e73016. doi:10.1371/journal.pone.0073016

Moffat, K. L., Kwei, A. S.-P., Spalazzi, J. P., Doty, S. B., Levine, W. N., and Lu, H. H. (2009). Novel Nanofiber-Based Scaffold for Rotator Cuff Repair and Augmentation. Tissue Eng. A 15 (1), 115-126. doi:10.1089/ten.tea.2008.0014

Morais, D. S., Cruz, J., Fangueiro, R., Lopes, H., Guedes, R. M., and Lopes, M. A. (2020). Mechanical Behavior of Ropes Based on Polypropylene (PP) and Poly(ethylene Terephthalate) (PET) Multifilament Yarns for Achilles Tendon Partial Substitution. J. Mech. Behav. Biomed. Mater. 106, 103734. doi:10.1016/j.jmbbm.2020.103734

Moshiri, A., Oryan, A., and Meimandi-Parizi, A. (2015). Synthesis, Development, Characterization and Effectiveness of Bovine Pure Platelet Gel-Collagen-Polydioxanone Bioactive Graft on Tendon Healing. J. Cel. Mol. Med. 19 (6), 1308-1332. doi:10.1111/jcmm.12511

Nguyen, T. P., Keyt, L. K., Herfat, S., Gordon, L., and Palanca, A. (2020). Biomechanical Study of a Multifilament Stainless Steel cable Crimp System versus a Multistrand Ultra-high Molecular Weight Polyethylene Polyester Suture Krackow Technique for Achilles Tendon Rupture Repair. J. Foot Ankle Surg. 59 (1), 86-90. doi:10.1053/ j.jfas.2019.01.022

Oryan, A., Moshiri, A., Parizi, A. M., and Maffulli, N. (2014). Implantation of a Novel Biologic and Hybridized Tissue Engineered Bioimplant in Large Tendon Defect: an In Vivo Investigation. Tissue Eng. Part. A. 20 (3-4), 447-465. doi:10.1089/ten.TEA.2013.0053

Ouyang, H. W., Goh, J. C. H., Mo, X. M., Teoh, S. H., and Lee, E. H. (2002). The Efficacy of Bone Marrow Stromal Cell-Seeded Knitted PLGA Fiber Scaffold for Achilles Tendon Repair. Ann. N Y Acad. Sci. 961 (1), 126-129. doi:10.1111/j.1749-6632.2002.tb03064.x

Ouyang, H. W., Goh, J. C. H., Thambyah, A., Teoh, S. H., and Lee, E. H. (2003). Knitted Poly-Lactide-Co-Glycolide Scaffold Loaded with Bone
Marrow Stromal Cells in Repair and Regeneration of Rabbit Achilles Tendon. Tissue Eng. 9 (3), 431-439. doi:10.1089/107632703322066615

Pietschmann, M. F., Frankewycz, B., Schmitz, P., Docheva, D., Sievers, B., Jansson, V., et al. (2013). Comparison of Tenocytes and Mesenchymal Stem Cells Seeded on Biodegradable Scaffolds in a Full-Size Tendon Defect Model. J. Mater. Sci. Mater. Med. 24 (1), 211-220. doi:10.1007/ s10856-012-4791-3

Reverchon, E., Baldino, L., Cardea, S., and De Marco, I. (2012). Biodegradable Synthetic Scaffolds for Tendon Regeneration. Muscles Ligaments Tendons J. 2 (3), 181-186.

Sahoo, S., Toh, S. L., and Goh, J. C. H. (2010). A bFGF-Releasing silk/PLGABased Biohybrid Scaffold for Ligament/tendon Tissue Engineering Using Mesenchymal Progenitor Cells. Biomaterials 31 (11), 2990-2998. doi:10.1016/j.biomaterials.2010.01.004

Sato, M., Maeda, M., Kurosawa, H., Inoue, Y., Yamauchi, Y., and Iwase, H. (2000). Reconstruction of Rabbit Achilles Tendon with Three Bioabsorbable Materials: Histological and Biomechanical Studies. J. Orthopaedic Sci. 5 (3), 256-267. doi:10.1007/s007760050161

Schoenenberger, A. D., Foolen, J., Moor, P., Silvan, U., and Snedeker, J. G. (2018). Substrate Fiber Alignment Mediates Tendon Cell Response to Inflammatory Signaling. Acta Biomater. 71, 306-317. doi:10.1016/ j.actbio.2018.03.004

Shieh, S.-J., Zimmerman, M. C., and Parsons, J. R. (1990). Preliminary Characterization of Bioresorbable and Nonresorbable Synthetic Fibers for the Repair of Soft Tissue Injuries. J. Biomed. Mater. Res. 24 (7), 789-808. doi:10.1002/jbm.820240702

Song, Z., Shi, B., Ding, J., Zhuang, X., Zhang, X., Fu, C., et al. (2015). A Comparative Study of Preventing Postoperative Tendon Adhesion Using Electrospun Polyester Membranes with Different Degradation Kinetics. Sci. China Chem. 58 (7), 1159-1168. doi:10.1007/s11426-015-5425-7

Song, Z.-m., Shi, B., Ding, J.-x., Zhuang, X.-l., Zhang, X.-n., Fu, C.-f., et al. (2015). Prevention of Postoperative Tendon Adhesion by Biodegradable Electrospun Membrane of Poly(lactide-Co-Glycolide). Chin. J. Polym. Sci. 33 (4), 587-596. doi:10.1007/s10118-015-1611-5

Vuornos, K., Björninen, M., Talvitie, E., Paakinaho, K., Kellomäki, M., Huhtala, H., et al. (2016). Human Adipose Stem Cells Differentiated on Braided Polylactide Scaffolds Is a Potential Approach for Tendon Tissue Engineering. Tissue Eng. Part. A. 22 (5-6), 513-523. doi:10.1089/ ten.tea.2015.0276

Wang, W., Deng, D., Wang, B., Zhou, G., Zhang, W., Cao, Y., et al. (2017). * Comparison of Autologous, Allogeneic, and Cell-free Scaffold Approaches for Engineered Tendon Repair in a Rabbit Model-A Pilot Study. Tissue Eng. Part. A. 23 (15-16), 750-761. doi:10.1089/ ten.TEA.2016.0447

Weng, C.-J., Lee, D., Ho, J., and Liu, S.-J. (2020). Doxycycline-Embedded Nanofibrous Membranes Help Promote Healing of Tendon Rupture. Int. J. Nanomedicine 15, 125-136. doi:10.2147/ijn.s217697

Yan, Z., Meng, X., Su, Y., Chen, Y., Zhang, L., and Xiao, J. (2021). Double Layer Composite Membrane for Preventing Tendon Adhesion and Promoting Tendon Healing. Mater. Sci. Eng. C 123, 111941. doi:10.1016/j.msec.2021.111941

Yildirim, Y., Saygi, B., Kara, H., Cabukoğlu, C., and Esemenli, T. (2006). Tendon Holding Capacities of the Suture Materials Used in Repairing Achilles Tendon Rupture. Acta Orthop. Traumatol. Turc. 40 (2), 164-168.

Yuan, B., He, C., Dong, X., Wang, J., Gao, Z., Wang, Q., et al. (2015). 5Fluorouracil Loaded Thermosensitive PLGA-PEG-PLGA Hydrogels for the Prevention of Postoperative Tendon Adhesion. RSC Adv. 5 (32), 25295-25303. doi:10.1039/c5ra01307k

Zhang, W., Yang, Y., Zhang, K., Li, Y., and Fang, G. (2015). Weft-knitted Silk-Poly(lactide-Co-Glycolide) Mesh Scaffold Combined with Collagen Matrix and Seeded with Mesenchymal Stem Cells for Rabbit Achilles Tendon Repair. Connect. Tissue Res. 56 (1), 25-34. doi:10.3109/ 03008207.2014.976309

Zhao, T., Qi, Y., Xiao, S., Ran, J., Wang, J., Ghamor-Amegavi, E. P., et al. (2019). Integration of Mesenchymal Stem Cell Sheet and bFGF-Loaded Fibrin Gel in Knitted PLGA Scaffolds Favorable for Tendon Repair. J. Mater. Chem. B 7 (13), 2201-2211. doi:10.1039/c8tb02759e 
Zou, J., Lu, M., Chen, S., Cai, C., Yao, Z., Cui, W., et al. (2020). Beeswaxinspired Superhydrophobic Electrospun Membranes for Peritendinous Anti-adhesion. Mater. Sci. Eng. C 116, 111166. doi:10.1016/ j.msec.2020.111166

Conflict of Interest: The authors declare that the research was conducted in the absence of any commercial or financial relationships that could be construed as a potential conflict of interest.

Publisher's Note: All claims expressed in this article are solely those of the authors and do not necessarily represent those of their affiliated organizations, or those of the publisher, the editors and the reviewers. Any product that may be evaluated in this article, or claim that may be made by its manufacturer, is not guaranteed or endorsed by the publisher.

Copyright $\odot 2022$ Zhang, Wang, Zhang, Yang and Zhang. This is an open-access article distributed under the terms of the Creative Commons Attribution License (CC BY). The use, distribution or reproduction in other forums is permitted, provided the original author(s) and the copyright owner(s) are credited and that the original publication in this journal is cited, in accordance with accepted academic practice. No use, distribution or reproduction is permitted which does not comply with these terms. 